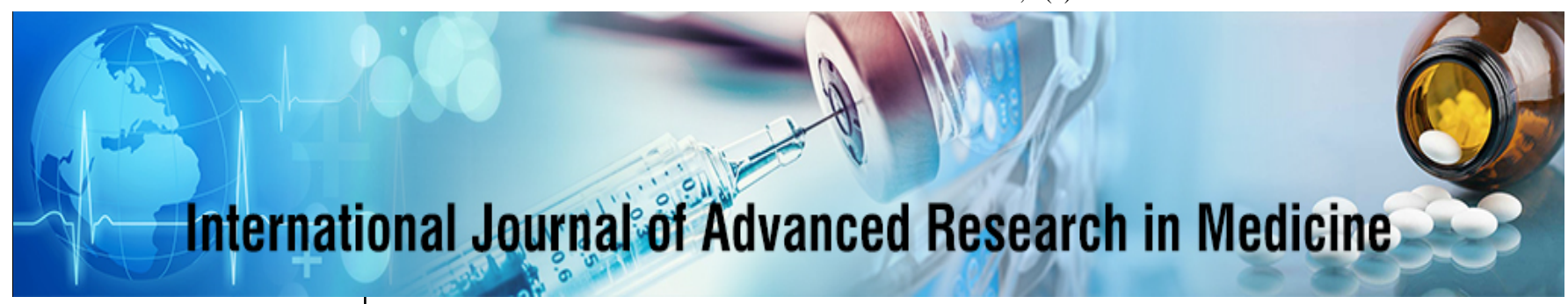

E-ISSN: 2706-9575

P-ISSN: 2706-9567

IJARM 2022; 4(1): 114-116

Received: 17-11-2021

Accepted: 21-12-2021

\section{Samer Talib}

Internal Medicine Department. Raritan Bay Medical Center at Perth amboy, New Jersey, USA
Corresponding Author: Samer Talib

Internal Medicine Department. Raritan Bay Medical Center at Perth amboy, New Jersey, USA

\section{Electronic cigarettes vaping impact on the respiratory system of vapers}

\section{Samer Talib}

DOI: https://doi.org/10.22271/27069567.2022.v4.i1b.347

\begin{abstract}
The use of E-cigarette or vaping has gained popularity in the past decade, especially among teenagers and young people, Also entering the pandemic era with people quarantining at home for long hours and mental health issues associated with this, the use of (ENDS) has gained even more popularity. In the summer of 2019(1), patients with respiratory illness related to vaping or ENDS use presented to healthcare facilities at an epidemic level that CDC labeled as (EVALI) till now there is no specific test, and diagnosis requires clinical, laboratory, with particular imaging findings. The emergence of the COVID pandemic and the overlapping imaging finding between conditions made EVALI diagnosis more challenging, especially with clinicians' approach focused more on COVID respiratory distress syndrome and overlooked EVALI in the differential diagnosis for respiratory distress syndrome. We present a case of respiratory distress with hypoxemia during the COVID pandemic her imaging finding overlapped with COVID and we focused mainly on COVID as a cause for this patient respiratory distress Then, COVID was ruled out as the culprit for this patient's symptoms and she was diagnosed with EVALI.
\end{abstract}

Keywords: EVALI, COVID, vaping, pandemic, ground glass opacity, confirmed case, probable case, vitamine e acetate, electronic nicotine delivery system(ends), e-cigarette.

\section{Introduction}

As of February 18, 2020, a total of 2,807 patients with EVALI have been reported to CDC [9]. Two thousand twenty-two of those hospitalized patients with Evali knew about their substance use, and $82 \%$ of them reported THC-containing products, with $33 \%$ confirmed exclusive THC products use ${ }^{[2,9]}$.

The street names of ENDS include (E-hookahs, vapes, tank systems, mods, vape pens, JUULUS, JUULING) all utilize a battery as a source of energy to produce an inhaled aerosol. When used, it converts a liquid to mist or vape users inhale; it has four main parts: ${ }^{[5}$, 8].

1. Rechargeable lithium battery.

2. The tank contains a liquid solution (e-liquid or $e$-juice) containing varying amounts of nicotine, flavorings, and other chemicals.

3. A heating coil (atomizer).

4. A mouthpiece (a consumer used to inhale) ${ }^{[5]}$.

The street name for the E-cigar liquid is Juice. Some E-cigar liquid contains propylene glycol or vegetable glycerin-based liquid with nicotine; other vaping liquid products contain THC and other toxic substances such as Vitamin E and CBD oil ${ }^{[2]}$. A Vaping liquid cig-alike brands contain chromium and nickel in high levels ${ }^{[5,6]}$. Dry herb vape devices heat dry Marijuana without combusting it and without using additional liquid. They are manufactured from quartz, ceramic, titanium ${ }^{[7]}$.

\section{Marijuana concentrates are produced commercially through:}

1. Dry processing.

2. Dry ice processing.

3. Water-based processing.

4. Combining pressure with heat.

5. Using nonflammable carbon dioxide solvents.

6. Using flammable solvents, butane, alcohol, propane, ether. 
Butane is a commonly used Solvents producing the potent Marijuana concentrate. Butane hash oil (BHO); $\mathrm{BHO}$ is very popular because of its high THC levels, longer-lasting effects, and low cost.

Case presentation: This is a 50 years old female who works from home with a past medical history significant for anxiety and marijuana medical use, who presented to ED with shortness of breath, and chest tightness; for the last four days patient endured fever (101.3), chills, nonproductive cough. ROS was unremarkable except as above. The patient denied a history of asthma or tobacco smoking, no other illicit drug use, no recent illness exposure, and is unvaccinated for COVID. The Patientreported recent Butane hash oil vaping.

In the ED, vitals (BP 126/59, heart rate 105, temperature 100.2, Spo2 on room air was 87, respiratory rate was 24 BPM. The patient was in distress; lung auscultation revealed basal rales; the patient was placed on 15 liters NRB to maintain Sp02 93\%. Labs showed WBC 18.1, eosinophil $0.0 \%$, bands $2.6 \%$, creatinine 0.8 , potassium 3.6 , arterial blood gas showed $\mathrm{pH} 7.4$,

CO2 35, O2 170 with 15 liters oxygen, D-dimer 411, troponin 0.01, procalcitonin was 0.14 , Streptococcal and legionella pneumonia antigens in urine were negative, mycoplasma antibody- negative also, respiratory pathogen panel was negative, COVID antigen and COVID antibody both were negative, HIV was negative, EKG was unremarkable. Chest x-ray showed bilateral infiltrates, CT Angio showed no pulmonary embolism, but extensive bilateral ground-glass pulmonary infiltrates. The patient was started on Prednisone $60 \mathrm{mg}$ daily and DUONEB (ipratropium bromide- Albuterol sulfate) breathing treatment, oxygen 15 liters per minute NRB. Later we added BREO (Fluticasone-vilanterol) then changed DUONEB to as needed with titration of oxygen to room air.

\section{Discussion: The use of ENDS is prevalent, and this is because}

1. The use of ENDS in the treatment of tobacco withdrawal and smoking cessation ${ }^{[10]}$.

2. A perception that ENDS is less harmful than cigarettes [10].

3. The evasion of exposing the household to secondhand smoking ${ }^{[10]}$.

4. Variations of flavors ${ }^{[11]}$.

5. Taste ${ }^{[11]}$.

6. Vaping for weight maintenance ${ }^{[12]}$.

Even though they are popular, they are poorly studied, and the pathogenesis remains poorly understood. A consistent pathological feature discovered in multiple pathological studies is the appearance of lipid-laden pulmonary alveolar macrophages, many with vacuolization and often with vacuolated pneumocytes-this is typical for chemicalinduced pneumonitis ${ }^{[2]}$. EVALI is associated with Vitamin $\mathrm{E}$ acetate, a constituent of THC vaping products found in Bronchoalveolar lavage fluids in patients with EVALI ${ }^{[1]}$; some other components and products may have pulmonary toxicity effects, as below ${ }^{[3,13]}$.

Product constituent (3) $\rightarrow$ Mechanism of lung injury Vitamin E constituent (1), (3) $\rightarrow$ Implicated in EVALI Atomizer (3) $\rightarrow$ Heavy metals toxicity

Flavoring (2-3) $\rightarrow$ Thermal degradation (Diacetyl, benzaldehyde)
Propylene glycol, vegetable glycerine $(3) \rightarrow$ Thermal degradation (Formaldehyde, acetaldehyde, lipid content).

General E. Cigar vapor (3) $\rightarrow$ Asthma, cough, Inflammatory changes, DNA Damage.

EVALI is a diagnostic dilemma, and there are no specific diagnostic tests; for definitive diagnosis, we need clinical, laboratory with imaging Data, suspect EVALI in a patient with constitutional, GI, or pulmonary symptoms with recent use of ENDS, no evidence of alternative diagnosis from history and physical exams, exclude infections by laboratory workup, chest imaging that identifies pulmonary infiltrate consistent with EVALI ${ }^{[14]}$.

CDC definitions for a confirmed and probable case of EVALI are intended for surveillance purposes and not for a clinical diagnosis ${ }^{[4]}$.

A confirmed case of EVALI must have --> Using an ecigarette ("vaping") or dabbing* in 90 days before symptom onset, and pulmonary infiltrates on chest X-ray or groundglass opacity on chest CT. Absence of pulmonary infection on initial workup:

1. A negative respiratory viral panel.

2. A negative influenza test by PCR or rapid test.

3. An unremarkable laboratory workup for all clinicallyindicated respiratory infectious disease testing (Urine antigen for Streptococcus pneumonia and Legionella).

4. Negative sputum culture if productive cough,

5. Negative blood culture,

6. HIV-related opportunistic respiratory infections are negative if there is evidence of HIV infection.

7. No evidence of plausible alternative diagnoses (e.g., cardiac, rheumatologic, or neoplastic process)

\section{A probable case must meet the following}

1. Use of (ENDS) or dabbing to inhale substances in 90 days preceding symptom onset.

2. Pulmonary infiltrate on imaging.

3. Either of infection identified by culture or PCR, but the clinical team believes this is not the sole cause of underlying lung injury minimum criteria to exclude pulmonary disease not met (testing not performed), but the clinical team thinks the infection is not the sole cause of underlying lung injury

4. No evidence of other differential diagnoses from history and physical exam.

Conclusions: E-cigarette use was labeled as pandemic among adults and youth in 2019; E- cigarette can cause extensive lung damage that can be fatal. The rise of the COVID pandemic led clinicians to focus their efforts on COVID and may have overlooked (ENDS). All healthcare providers need to be vigilant and inquire about vaping history. Also, all patients must be counseled on ENDS use and educated on the related health risks. Patients should be strongly discouraged.

\section{References}

1. Vitamin E Acetate in Bronchoalveolar-Lavage Fluid Associated with EVALI, Benjamin C Blount, et al., NEJM

2. Vaping-induced acute lung injury, David C Christiani, NEJM.

3. innicka L, Shenoy MA. EVALI and the Pulmonary Toxicity of Electronic Cigarettes: A Review. J Gen Intern Med. 2020 Jul;35(7):2130-2135. DOI: 
10.1007/s11606-020-05813-2. Epub 2020 April 3. PMID: 32246394; PMCID: PMC7351931.

4. 4-https://www.cdc.gov/tobacco/basic_information/ecigarettes/assets/2019-Lung-Injury- Surveillance-CaseDefinition-508.pdf

5. Vaping Devices (Electronic Cigarettes) DrugFacts. National Institute on Drug Abuse website. https://www.drugabuse.gov/publications/drugfacts/vapi ng-devices-electronic-cigarettes.

6. Salzman GA, Alqawasma M, Asad H. Vaping Associated Lung Injury (EVALI): An Explosive United States Epidemic. Mo Med. 2019;116(6):492-496.

7. NIDA. Marijuana Concentrates DrugFacts. National Institute on Drug Abuse website. https://www.drugabuse.gov/publications/drugfacts/mari juana-concentrates.

8. www.dea.gov > sites > default > files > Vaping

9. https://www.cdc.gov/tobacco/basic_information/ecigarettes/severe-lung-disease.html\#latest- information

10. http://pubmed.ncbi.nlm.nih.gov/27914771?dopt=Abstra ct

11. http://pubmed.ncbi.nlm.nih.gov/29882828?dopt=Abstra cthttp://pubmed.ncbi.nlm.nih.gov/27875790?dopt=Abst ract

12. http://pubmed.ncbi.nlm.nih.gov/31577870?dopt=Abstra ct

13. MMWR Morb Mortal Wkly Rep. 2019 October 18; 68:919-927

14. McAlinden KD, Eapen MS, Lu W, Sharma P, Sohal SS. The rise of electronic nicotine delivery systems and the emergence of electronic-cigarette-driven disease. Am J Physiol Lung Cell Mol Physiol. 2020;319(4):L585L595. Doi:10.1152/ajplung.00160.2020.

15. Ansari-Gilani K, Petraszko AM, Gilkeson RC. COVID19 pneumonia versus EVALI, distinguishing the overlapping CT features in the COVID-19 era. Heart Lung. 2020;49(6):885-886. doi:10.1016/j.hrtlng.2020.06.008 